

\title{
Réponse à Alain Prochiantz et à sa biologie des mauvaises humeurs
}

A. Prochiantz a de mauvaises humeurs vis-à-vis des - comment appelle-t-il cela ? - des «frustrés-grincheux", des mauvais physiologistes, des intégristes de la complexité qui ne songeraient qu'à prendre leur revanche sur les bons molécularistes si gentils qui eux respectent et lisent l'œuvre de Claude Bernard.

Nous en sommes fort aise; «nous» étant mis pour un modeste représentant de ceux qui se réjouissent qu'enfin un peu de pensée physiologique saupoudre très finement le réductionnisme forcené de ces dernières années. Je n'irai pas jusqu'à rallier le camp des lobbyistes de l'anti-molécule mais «la physiologie revient» et la joie revancharde de quelques-uns n'a rien de bien étonnant eu égard au couvercle pesant que le clan des pro-molécules - quasiment atomique - fait peser. Depuis trop longtemps, les pires âneries énoncées avec la force que la loi moléculaire et cellulaire leur conférait nous submergent; on guérit le cancer trois ou quatre fois par semaine; on prédit l'immortalité pour demain matin, le séquençage de je-ne-sais-quel-gène a vaincu l'obésité, les troubles du sommeil et permet même de guérir des écrouelles et demain matin la structure des quarks bouleversera la pharmacolo- gie. Beaucoup n'hésitent pas à tirer sur la corde, leur démesure mérite bien nos moqueries.

Les instructions viennent de loin ; les tenants de l'intégré peuvent sortir de la marginalisaton. Devant la situation actuelle, ceux-ci alternent entre le rire sarcastique et l'indignation; les crédits pleuvent pour construire des animaleries, qu'on leur refusait, remplies de souris transgéniques dont personne ne sait que faire; on découvre que l'inactivation de ce gène, si important dans une cellule en culture, n'induit aucune manifestation sur un organisme, on redécouvre que le tout n'est pas que la somme des parties, que de puissants mécanismes de compensation existent dans l'organisme et même que c'est bien la caractéristique des êtres vivants ; ils s'adaptent.

Faisons fi des querelles partisanes, j'ai bien peur que ce mouvement d'humeur d'A. Prochiantz ne cache des problèmes de fond plus sérieux. Il existe, c'est vrai, des "grincheux », heureux d'arracher un peu de pouvoir aux sciences mols, comme il existe, c'est absolument sûr, des «réductionnistes pleins de bonne volonté» qui ne se sont pas privés pour ramener à rien la physiologie. Le renouveau se manifeste, on parle de physiologie. Il ne s'agit plus de savoir qui fait de la bonne physiologie mais plutôt de savoir si la vision "physiologie» va enfin servir de guide, de fil conducteur ou si on se contentera de créditer quelques équipes chargées de porter bien haut un étendard de parade.

A mon sens, il n'est plus temps de s'interroger sur les vrais détenteurs de la pensée bernardienne, il est plus urgent de trouver des physiologistes. Cette «race-là» a quasiment disparu; les plus tenaces sont partis mesurer les fréquences cardiaques des manchots empereurs et les plus malins ont drapé leurs activités dans un outillage moléculaire qui a trompé tout le monde mais d'où il ne sont pas sortis. Les bailleurs de fonds ont commencé à comprendre que cloner n'était pas guérir, que séquencer n'était pas comprendre; une fois les séquences du génome humain, murin, bactérien alignées, une fois clos le répertoire des protéines possibles et des éléments cellulaires de régulation probables, il va falloir se mettre au travail et rendre compte de l'activité intégrée de l'ensemble de tous ces éléments et de leur interaction produisant une fonction plus globale. La domination d'une science par une autre n'est pas à l'ordre du jour; prétendre assujettir la génétique, la biologie cellulaire à 
la physiologie ou le contraire ne sert qu'à justifier des attributions arbitraires de crédits; il sera plus utile de rassembler nos connaissances de l'élémentaire pour appréhender le fonctionnement intégré de l'organisme qui lui, en tant qu'humain, nous intéresse au plus haut point.

Cela nécessite de nouveaux chercheurs, maîtrisant certes les indispensables outils moléculaires, mais sur- tout imprégnés par les paradigmes de la physiologie et doués de quelques connaissances des grandes fonctions. En tout cas, des chercheurs qui se situent au-delà de ces guerres picrocholines qui opposent la complexité de l'intégré à la naïveté du réduit. Il serait plus utile maintenant de commencer à rassembler des forces créatrices et non d'opposer des exégèses de la pensée de C. Bernard dont l'aspect pratique a perdu de sa valeur aujourd'hui

\section{Jean-Marie Zajac}

Institut de pharmacologie et de biologie structurale, Cinrs, 205, route de Narbonne, 31077 Toulouse Cedex, France.

\section{Le Club NO}

Une association dite Club NO a été créée en 1993 pour échanger les compétences et les informations détenues par des scientifiques des secteurs public et privé, au sujet de la biosynthèse et des propriétés du NO. NO n'est pas un sigle, ni un signe, c'est la formule du monoxyde d'azote, gazeux à l'état pur. Sa structure électronique indique que c'est un radical stable dans les conditions normales de température et de pression. Cette propriété régit sa réactivité. Produit de départ de l'industrie chimique lourde, composé polluant des gaz de l'atmosphère urbaine parce que source d'oxydes toxiques, mais aussi produit de la dénitrification bactérienne dans les eaux et le sol, il est enfin apparu comme étant un composé que fabriquent la plupart des animaux et l'homme à partir d'un acide aminé essentiel: la L-arginine, et intervenant dans les grandes fonctions physiologiques.

Dans le numéro de novembre de 1998 de médecine/science, Anh Tuan Dinh-Xuan a analysé les motivations qui avaient conduit l'Académie Royale de Suède à décerner le prix Nobel de Médecine et Physiologie 1998 à Robert Furchgott, Louis Ignarro et Ferid Murad, et décrit l'historique des recherches sur le "monoxyde d'azote (NO) en tant que molécule de signalisation dans le système cardiovasculaire ». Ce sont leurs travaux au début des années 1980, sur les agents dilatateurs des vaisseaux sanguins qui ont conduit à la découverte du rôle du NO. Il a néanmoins fallu attendre 1988 pour que Salvador Moncada, John Hibbs et Michael Marletta, indépendamment, établissent que NO provient de la L-arginine, ce qui a alors ouvert un champ nouveau à la biologie.

NO a connu ensuite une ascension vers le cerveau lorsqu'il fut démontré que des cellules nerveuses pouvaientelles aussi le produire. C'est en effet un neurotransmetteur dans le système nerveux central et périphérique. Grâce à ses propriétés de diffusion, il peut servir de rétromessager entre les neurones qui l'utilisent pour stocker des informations au cours de l'apprentissage et de la formation de la mémoire.

NO est donc un messager des interactions cellulaires et un agent effecteur dans toutes les fonctions biologiques. Ce rôle ubiquitaire vient de l'existence de trois enzymes différentes capables de synthétiser $\mathrm{NO}$, les NO-synthases, qui ont chacune leur distribution et leur finalité. Ainsi, le NO produit par la NO-synthase endothéliale exerce un tonus vasodilatateur permanent, participant au maintien de la pression sanguine artérielle. Sa production accrue en réponse à l'augmentation du débit sanguin permet une adaptation du calibre artériel afin de répondre précisément aux besoins tissulaires. Notons que de nombreuses affections touchant la paroi artérielle (comme l'athérosclérose) résultent d'anomalies de la vasodilatation dépendante de l'endothélium. Mais on retrouve finalement des dys- fonctionnements liés à la production dérégulée de NO dans tous les systèmes physiologiques. D'où l'intérêt de favoriser les recherches visant à mieux contrôler l'activité des NOsynthases par l'obtention d'inhibiteurs spécifiques de chacune d'elles. A l'inverse, il sera utile de disposer de donneurs de NO ciblés. Un autre axe de recherche s'efforcera d'identifier toutes les cibles moléculaires de NO et de contrôler les voies de signalisation qui leur sont liées.

Les membres du Club NO sont particulièrement heureux de l'attribution du prix Nobel à la molécule qui les passionne et les rassemble. NO est l'un des effecteurs biologiques les plus petits par la structure chimique, mais probablement l'un des plus importants par l'universalité de ses fonctions dans les processus de la vie. Il mène un jeu subtil avec l'oxygène, les radicaux et les métaux de transition. Des physiciens, chimistes, biologistes physiologistes et médecins ont décidé depuis plusieurs années de s'unir pour en comprendre les implications dans tous les domaines de la santé

Serge Adnot, Jean-François Arnal, Andrees Böhme, Jean-Luc Boucher, Pierre-Étienne Chabrier, Anh Tuan Dinh-Xuan, Jean-Claude Drapier, Claire Ducrocq, Olivier Goureau, Annie Guissani, Yann Henry, Michel Lepoivre, Jean-Christophe Mercier, Yvette Morot-Gaudry, Bernard Muller, Jean-François Petit, Michel Plotkine, Jean-Claude Stoclet. Club NO, Institut Curie, Inserm U. 350, UPS, bâtiment 112, 91405 Orsay, France. http://www.curie.u-psud.fr/ClubNO/ 